\title{
Investigating the performance of catalyst layer micro-structures with different platinum loadings
}

\author{
M. Khakaz-Baboli ${ }^{\mathrm{a}}$, D.A. Harvey ${ }^{\mathrm{a}, \mathrm{b}}$, J.G.Pharoah ${ }^{\mathrm{a}}$, \\ ${ }^{a}$ Queen's-RMC Fuel Cell Research Centre, Queen's University, Kingston, ON, K7L3N6, \\ CANADA \\ ${ }^{\mathrm{b}}$ Ballard Power Systems, 9000 Glenlyon Parkway, Burnaby, BC, V5J 5J8, CANADA
}

In this study a four-phase micro-structure of a PEFC catalyst layer was reconstructed by randomly placing overlapping spheres for each solid catalyst phase. The micro-structure was mirrored to make a $1 \mu \mathrm{m}$ micro-structure. A body-fit computational mesh was produced for the reconstructed micro-structure in OpenFOAM. Associated conservation equations were solved within all the phases with electrochemical reaction as the boundary condition at the interface between ionomer and platinum phases. The study is focused on the platinum loading of CL. The polarization curves of the micro-structure performance have been compared for different platinum loadings. This paper gives increased insight into the relatively greater losses at decreased platinum loadings.

\section{Introduction}

Catalyst layers with low platinum loadings are more commonly used to reduce the production cost. While very low platinum loadings are desired, it is important to retain good catalyst performance as the loading is decreased. The micro-scale phenomena in catalyst layer are not yet fully understood. For instance, It has been observed that the resistance in oxygen transport unexpectedly increases at very low platinum loadings in comparison to high platinum loadings, $(1,2)$. This has been modelled using an agglomerate model of a proton exchange membrane catalyst layer (3) and it was found that the ionomer film thickness covering the agglomerates causes mass transport resistance which increases at very low platinum loadings. This makes it necessary to understand the micro-scale phenomena within catalyst layers. Recently, micro-modelling of the catalyst layer in proton exchange membrane has drawn increased attention. In these studies, a micro-structure of the catalyst layer is reconstructed and the conservation equations are solved within each constituent (4), (5).

\section{Micro-structure reconstruction and grid generation}

The CL micro-structure was reconstructed by pseudo-randomly placing spheres of constituents phases; carbon, platinum and ionomer (6). Spheres of one constituent are overlapped so that a percolating phase is formed for the sake of transport throughout the micro-structure. The interface between the four phases are captured and boundary conditions are to be set these interfaces. The reaction is presumed to occur only on the interface between the ionomer phase and the Pt phase. The initial micro-structure was produced within a cube of dimension $100 \mathrm{~nm}$, which is repeatedly mirrored in order to generate a $1 \mathrm{~mm}$ thick catalyst layer as shown in Figure 1. 

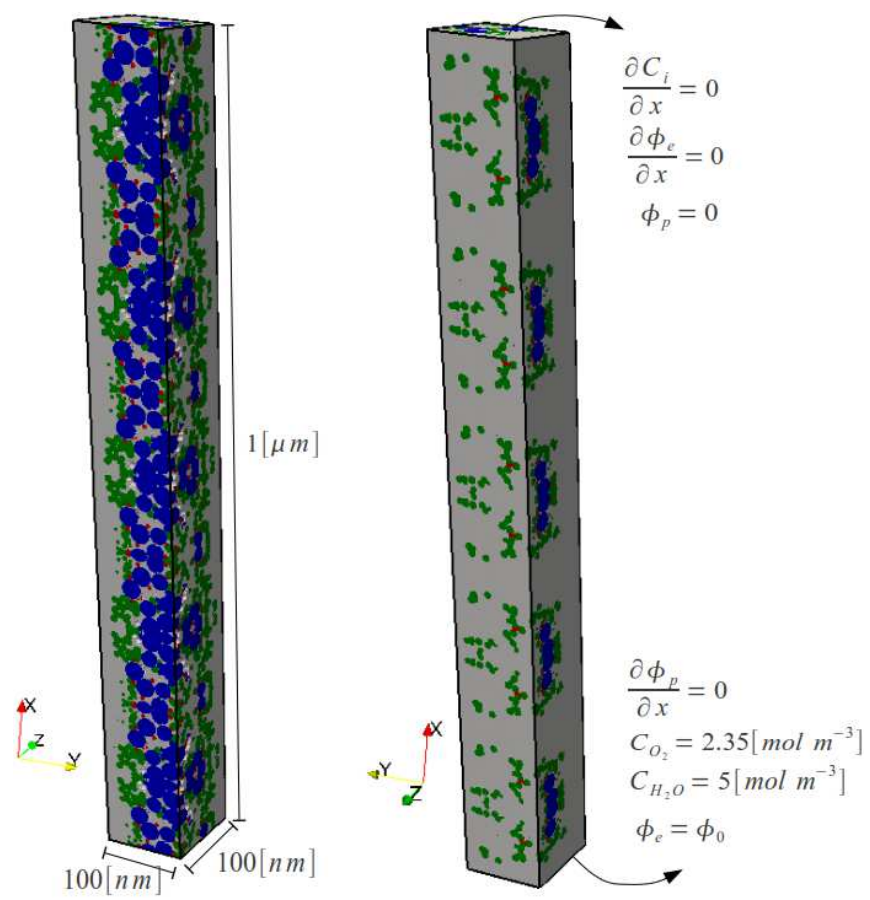

Figure 1. Left: the four-phase microstructure of with pore phase in gray, carbon phase in blue, ionomer phase in green and platinum phase in red. Right: the boundary conditions used for numerical modeling.

The highest platinum loading of $0.6 \mathrm{mg} / \mathrm{cm}^{2}$ was used to produce the micro-structure, using $50 \% \mathrm{Pt}$ on $\mathrm{C}$ with an ionomer loading of $23 \%$. The porosity of the micro-structure is 0.49 which corresponds to a $12 \mu \mathrm{m}$ thick catalyst layer. Due to the large computational requirements, the actual simulated domain is only $1 \mu \mathrm{m}$ thick and as such the Pt loadings on the actual simulated domains are $1 / 12$ of the reported loading for a full thickness. The platinum loading of the micro-structure was decreased by randomly removing platinum particles by considering them to be made of carbon. This has been done to produce three more micro-structures with platinum loadings of $0.4,0.3$ and $0.1 \mathrm{mg} / \mathrm{cm}^{2}$. Figure 2 depicts slices through the mid-section of the catalyst sample for each Pt loading. 

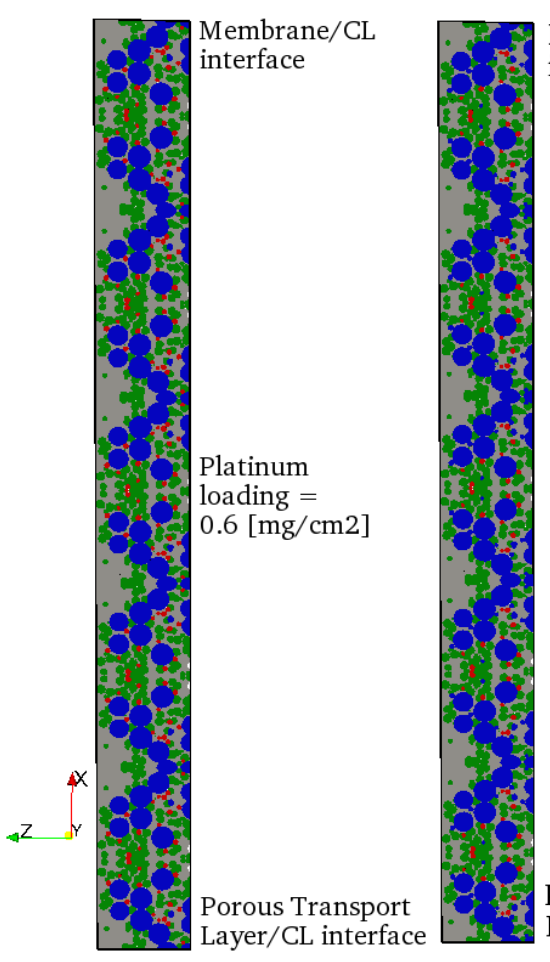

Membrane/CL interface

Platinum loading $=$ $0.4[\mathrm{mg} / \mathrm{cm} 2]$
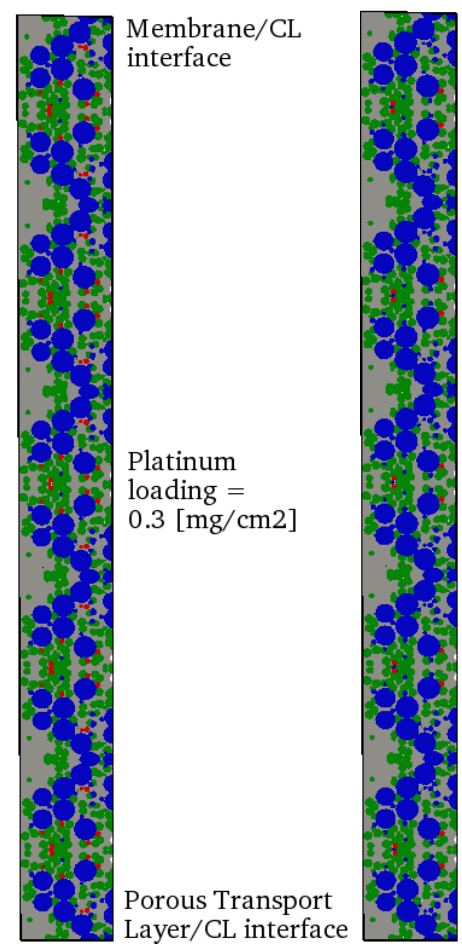

Membrane/CL interface Layer/CL interface

Platinum loading $=$ $0.1[\mathrm{mg} / \mathrm{cm} 2]$

Porous Transport Layer/CL interface

Figure 2. Slices of micro-structures with different platinum loadings produced by randomly excluding platinum particles and adding them to carbon phase.

\section{Governing Equations}

Conservation of mass considering diffusive transport has been used to model species transport in pore phase of the micro-structure:

$$
\nabla \cdot\left(D_{i}^{e f f} \nabla y_{i}\right)=0
$$

where $D_{i}^{e f f}$ is the effective diffusivity including Knudsen effects:

$$
\frac{1}{D_{i}^{e f f}}=\frac{1}{D_{K n}}+\frac{1}{D_{i b}}
$$

where $D_{i b}$ is the bulk diffusivity of species $i$. The same equation has been used to model species transport through the ionomer region, using a constant diffusivity with no correction for Knudsen effects.

Henry's law was implemented on the interface between pore and ionomer regions:

$$
\left.y_{\mathrm{O}_{2}}\right|_{\text {ionomer }}=\frac{\left.p_{\mathrm{O} 2}\right|_{\text {pore }}}{H}
$$

Conservation of charge was used to model positive charge transport though ionomer phase of micro-structure:

$$
\nabla \cdot\left(-\sigma_{p} \nabla \varphi_{p}\right)=0
$$

Conservation of charge was also implemented within the electronically conducting phase:

$$
\nabla \cdot\left(\sigma_{e} \nabla \varphi_{e}\right)=0
$$


The Butler-Volmer equation was employed to model surfacial electrochemical reaction on the interface between ionomer and platinum phases:

$i=i_{0} \frac{C_{O_{2}}^{\Gamma}}{C_{O_{2}}^{r e f}}\left[\exp \left(-\frac{n \alpha_{c} F}{R T} \eta^{\Gamma}\right)-\exp \left(\frac{n\left(1-\alpha_{c}\right) F}{R T} \eta^{\Gamma}\right)\right], \eta^{\Gamma}=\varphi_{e}^{\Gamma}-\varphi_{p}^{\Gamma}-E_{e q}[6]$

where $\Gamma$ indicates the value of variables on the interface between ionomer and platinum phases. The boundary conditions on the outer surface of the microstructure is illustrated in Figure 1. $\psi_{e}$ was set to different values at the bottom in order to polarize the electrode, while $\psi_{p}$ was taken as zero on the membrane catalyst interface. The input parameters including the operating conditions and transport properties are shown in table I. The Open source CFD package OpenFOAM was used to solve the coupled set of equations.

TABLE I. Transport properties and operating conditions

\begin{tabular}{|c|c|c|c|}
\hline$D_{O 2, b}$ & $5.8 \times 10^{-6} \mathrm{~m}^{2} / \mathrm{s}$ & $\sigma_{\mathrm{p}}$ & $10 \mathrm{~S} / \mathrm{m}$ \\
\hline$D_{H 2 O, b}$ & $7.5 \times 10^{-6} \mathrm{~m}^{2} / \mathrm{s}$ & $\sigma_{\mathrm{e}}$ & $1000 \mathrm{~S} / \mathrm{m}$ \\
\hline$D_{O 2, \text { ionomer }}$ & $8.45 \times 10^{-10} \mathrm{~m}^{2} / \mathrm{s}$ & $\mathrm{H}$ & $0.3125 \mathrm{~m}^{3} \mathrm{~atm} / \mathrm{mol}$ \\
\hline$i_{0}$ & $4.5 \times 10^{-4} \mathrm{~A} / \mathrm{m}^{2} \mathrm{Pt}$ & $n$ & 2 \\
\hline$C_{O_{2}}^{\text {ref }}$ & $41.59 \mathrm{~mol} / \mathrm{m}^{3}$ & $\alpha_{\mathrm{c}}$ & 0.49 \\
\hline$p$ & $1.3 \mathrm{~atm}$ & $\mathrm{~T}$ & $343.15 \mathrm{~K}$ \\
\hline$x_{\mathrm{O} 2}$ & $5 \%$ & $\mathrm{E}_{\mathrm{eq}}$ & $1.23 \mathrm{~V}$ \\
\hline
\end{tabular}

\section{Results}

Figure 3 depicts the local current density in each case as a function of distance through the catalyst layer. In all cases, there is current generation throughout the layer, but for the higher loadings there is relatively more current produced adjacent to the membrane/catalyst interface, and this illustrates the importance of proton transport within the catalyst layer. 


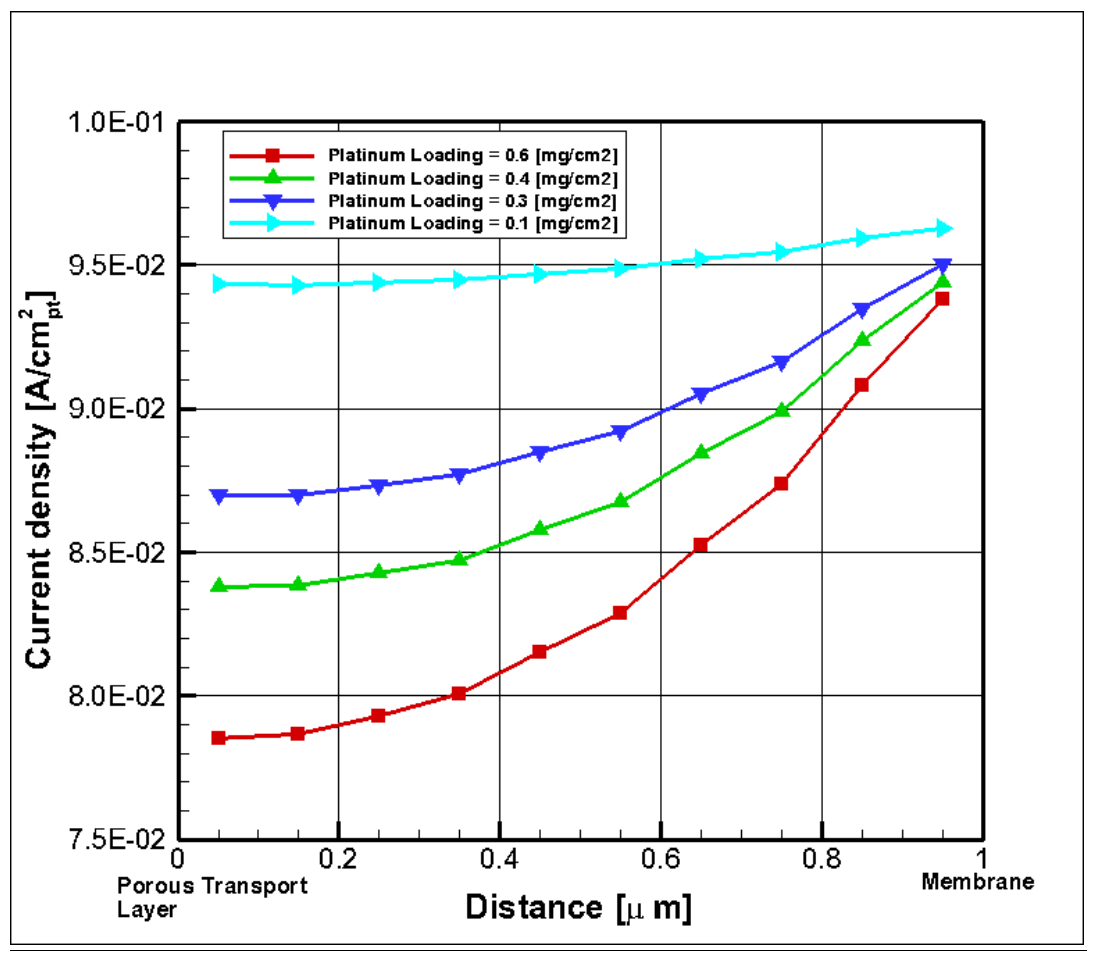

Figure 3. Local current density along the microstructure

Figure 4 shows the ionic potential within the ionomer phase for each of the four $\mathrm{Pt}$ loadings. At the highest Pt loading, the current is almost an order of magnitude higher than in the case of the lowest loading and there is a corresponding drop of approximately $6.5 \mathrm{mV}$ over the catalyst layer. At the lowest Pt loading, the drop in ionic potential over the layer is of the order of $1 \mathrm{mV}$ and the current generation is uniform throughout the entire layer. It is important to note that the ionic conductivity used for these simulations corresponds to the bulk value for fully humidified Nafion while it is quite likely that the ionic conductivity in the catalyst layer is lower than this. 


\begin{tabular}{|c|c|c|c|c|}
\hline 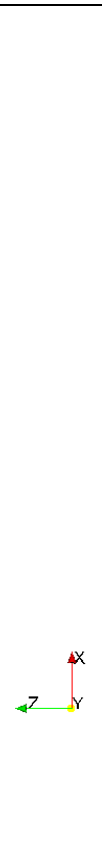 & 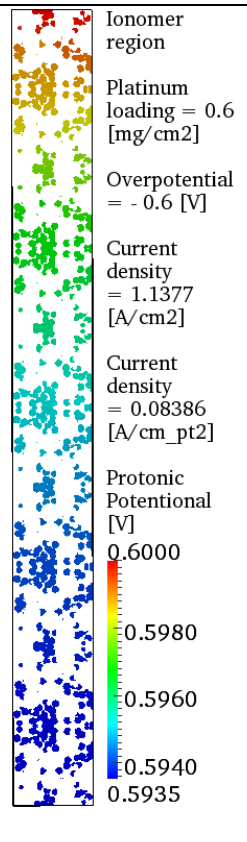 & 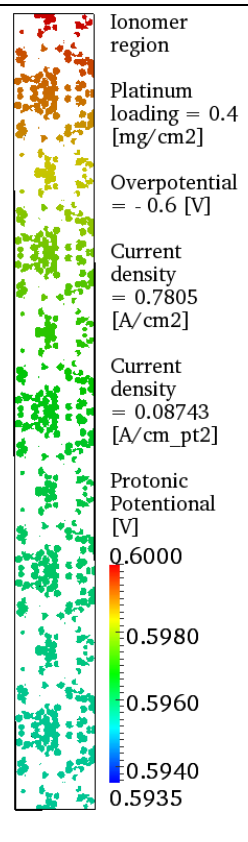 & 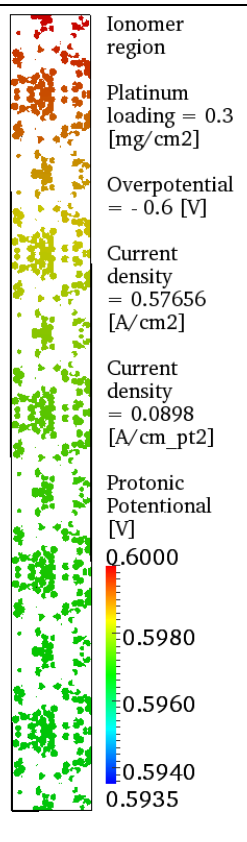 & $\begin{array}{lll} & \text { Ionomer } \\
\text { region } \\
\end{array}$ \\
\hline
\end{tabular}

Figure 4. Ionic potential in the ionomer phase for each Pt loading on a plane in the middle of the modeled catalyst layer. $\mathrm{x}$ is the dimension through the catalyst layer thickness, with $\mathrm{x}=0$ corresponding to the interface with the membrane.

Figure 5 depicts the oxygen concentration in the ionomer phase, while Figure 6 shows the gas phase oxygen concentration. The low relative concentration in the ionomer reflects the application of Henrys law. In the gas phase, there is a relatively uniform concentration gradient from the edge of the catalyst layer towards the membrane, and this gradient increases with increasing current density (and Pt loading). It is clear from Figure 5, that there is very strong local depletion in the vicinity of the Pt particles, and this is most evident at the lowest Pt loading as the gas phase gradient superimposed on ionomer distribution is the lowest. There is a relative increase in the local loss across the thickness of the ionomer as the Pt loading is decreased, as the current density relative to the platinum surface area increases with decreased Pt loading (shown on the figures) meaning that the oxygen flux through the ionomer is increasing at a given Pt site as the loading decreases. 


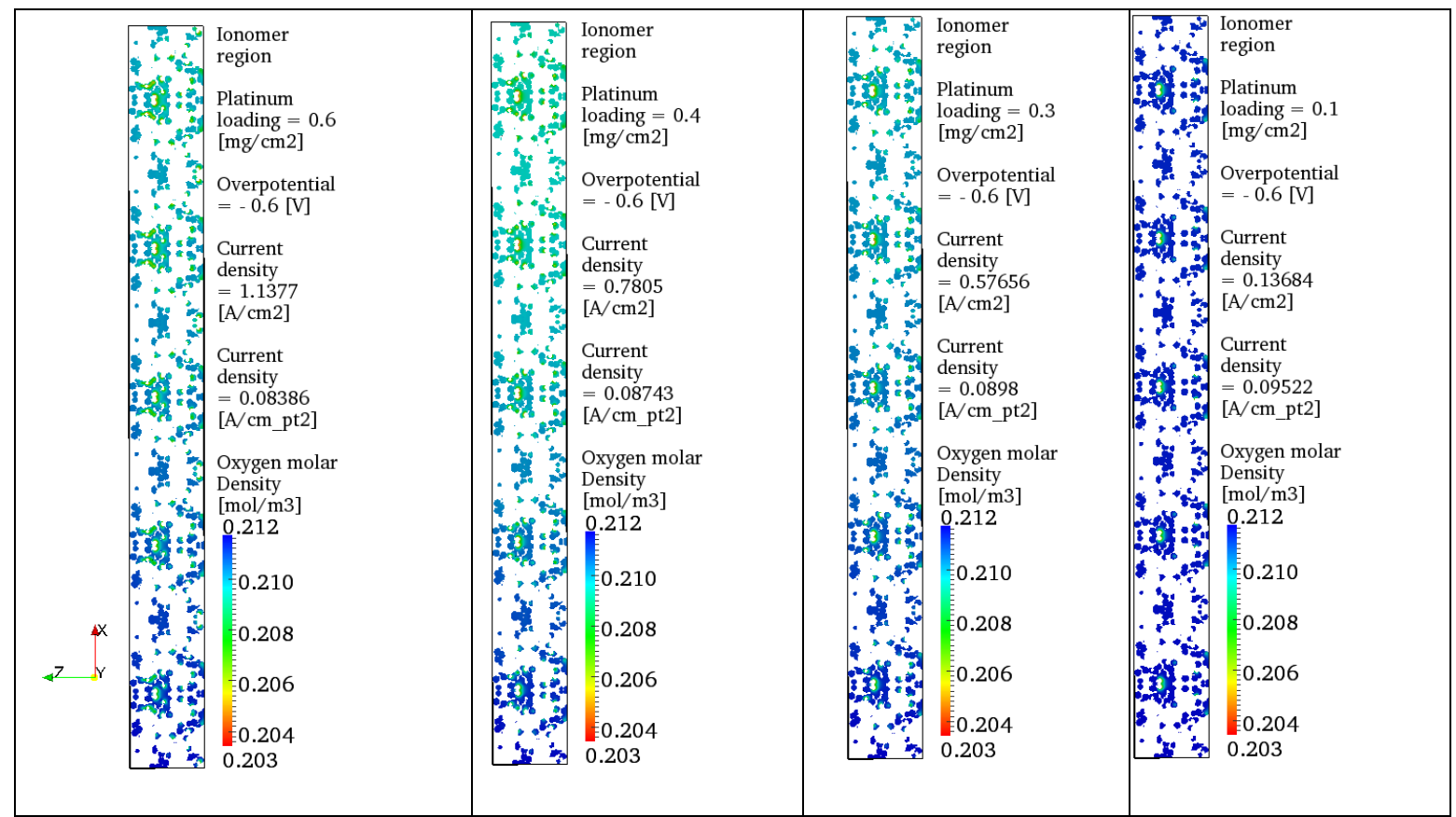

Figure 5. Oxygen concentration in the ionomer phase for each Pt loading on a plane in the middle of the modeled catalyst layer. $\mathrm{x}$ is the dimension through the catalyst layer thickness, with $\mathrm{x}=0$ corresponding to the interface with the membrane.

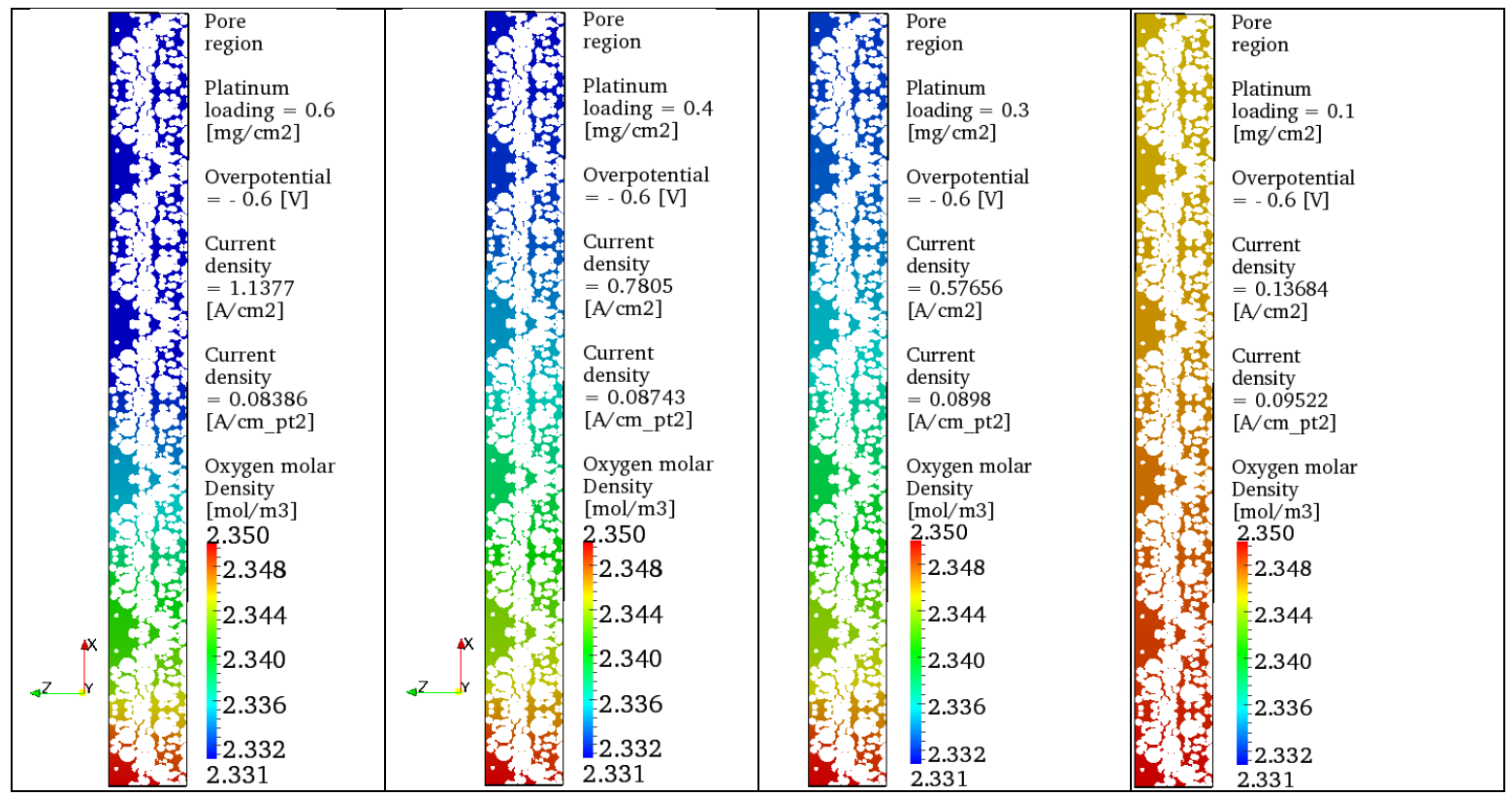

Figure 6. Oxygen concentration in the gas phase for each Pt loading on a plane in the middle of the modeled catalyst layer. $\mathrm{x}$ is the dimension through the catalyst layer thickness, with $\mathrm{x}=0$ corresponding to the interface with the membrane. 


\section{Conclusions}

A numerical model has been developed to study the detailed transport and kinetics on micro-structural representations of a catalyst layer. The model has been applied to a $1 \mu \mathrm{m}$ thick catalyst layer with 4 different Pt loadings which were achieved by randomly changing Pt particles to carbon particles such that the base geometries were virtually identical for each Pt loading. It was found that both the transport of protons and oxygen were significant for the resulting performance, with increased local losses in the ionomer at reduced Pt loading. When the catalyst is highly loaded, the current production is relatively greater adjacent to the membrane interface, while at lower currents the current production is virtually uniform.

\section{Acknowledgments}

Project funding by Ballard Power Systems is gratefully acknowledged.

\section{References}

1. N. Nonoyama, S. Okazaki, A. Z. Weber, Y Ikogi, and T. Yoshida, J. Electrochem. Soc., 158, B416 (2011).

2. H. A. Gasteiger, J. E. Panels, and S. G. Yan, J. Power Sources, 127, 162 (2004).

3. W. Yoon, and A. Z. Weber, J. Electrochem. Soc., 158(8), B1007 (2011).

4. N. A. Siddique and F. Liu, J. Electrochimica Acta, 55, 5357 (2010).

5. K. J. Lange, P. C. Sui, and N. Djilali, J. Power Sources, 196, 3195 (2011).

6. J.G. Pharoah, H-.W. Choi, C-.C. Chueh, D.A. Harvey, ECS Trans., 41(1), pp 221-227 (2011). 